\title{
The LHCb VELO: Status and Upgrade Developments
}

\author{
A G Bates ${ }^{a, b}$ on behalf of the LHCb VELO group \\ ${ }^{a}$ CERN, CH-1211, Geneva 23, Switzerland. \\ ${ }^{b}$ The University of Glasgow, Dept. of Physics and Astronomy, Glasgow, G12 8QQ.
}

\begin{abstract}
The LHCb VErtex LOcator (VELO) is a silicon based vertexing sub-detector which has active silicon positioned only $8 \mathrm{~mm}$ from the LHC beams and will operate in an extreme (up to $1.3 \times 10^{14} 1 \mathrm{MeV}$ neutron equivalents $/ \mathrm{cm}^{2} /$ year) and non-uniform radiation environment. The complex design of the VELO silicon sensors exploits oxygenated silicon and $n^{+}$-on- $n$ technology. Research has been carried out into new materials which could significantly extend the lifetime of silicon detectors at the LHC, these would have particular application in a VELO upgrade. Promising new results on the first test beam of a large, high resistivity Czochralski silicon detector with $50 \mu \mathrm{m}$ pitch and $40 \mathrm{MHz}$ electronics will be presented. The performance was studied before and after irradiation with high energy protons. A signal to noise of over $20: 1$ was obtained from the detector and after a fluence of $4.3 \times 10^{14} 1 \mathrm{MeV} \mathrm{n}_{e q}$ significant charge collection efficiencies were measured at relatively modest voltages. Studies using the Transient Current Technique probed the electric field within $\mathrm{MCz}$ test detectors and proved that $\mathrm{MCz}$ silicon does not type invert up until a radiation level of at least $5 \times 10^{14} 24 \mathrm{GeV} / \mathrm{c} \mathrm{p} / \mathrm{cm}^{2}$. This would mean the VELO could replace, in a potential upgrade, $\mathrm{n}^{+}$. on-n DOFZ sensors and the complicated processing involved, for standard $\mathrm{p}^{+}$-on-n processing with $\mathrm{MCz}$ - if $\mathrm{MCz}$ sensors prove to be sufficiently radiation hard.
\end{abstract}

IEEE International Conference, Nuclear Science Symposium in Rome, Italy 


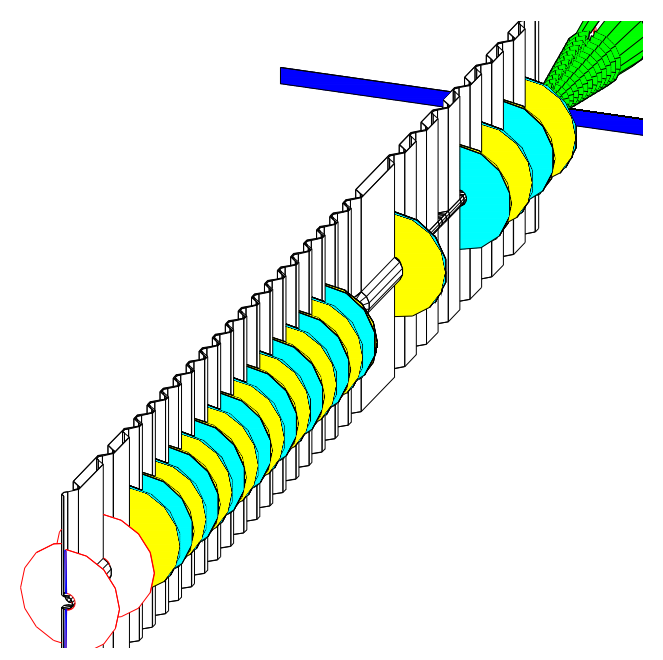

Figure 1: The 21 semi-circular VELO silicon stations positioned around the LHC beams. The sensors are separated from the primary LHC vacuum by a thin RF foil. The RF foils has 3 purposes: to protect the VELO form the beam, to protect the beam from the VELO and to guide the wake fields.

\section{Introduction}

The LHCb detector is a dedicated B-sector CP violation experiment for the LHC collider. The detector is a single arm spectrometer with an angular acceptance of $15-300 \mathrm{mrad}$. The detector contains two silicon tracking systems: the inner tracker and the VErtex LOcator (VELO). The tasks of the VELO include the reconstruction of the production and decay vertices of b-hadrons, necessary to distinguish between signals from b-hadron decays and background. Moreover the VELO has to reconstruct tracks covering the full angular acceptance of the downstream detectors and provide information to the second level trigger. The VELO makes heavy demands on the silicon performance, due to its participation in the trigger and the importance of making measurements in the rapidly oscillating $\mathrm{B}_{s}$ channels.

The VELO will be described in more detail in section 2. The sensor design and detector construction will be briefly outlined and test beam results on the last proto-type detector will be presented. $\mathrm{LHCb}$ is due to commence data taking in Spring 2007. Due to the extremely harsh and non-uniform LHCb radiation environment, the current sensors are expected to require replacement after 3 years of operation. A possible upgrade choice is suggested in section 3 and test beam and transient current technique results give promising information for this upgrade option.

\section{VErtex LOcator}

\subsection{Sensor Design}

Obtaining the optimal impact parameter resolution requires a short extrapolation distance to the vertex as well as a minimum of material before the first measured point. This is achieved by placing the sensors as close as possible to the LHC beams. Hence, the VELO consists of 21 stations which are perpendicular to the LHC beams over a distance of $1 \mathrm{~m}$ around the interaction region, as shown in Fig. 1 . The first active silicon strip is only $8 \mathrm{~mm}$ from the LHC beams. This results in an extreme, non-uniform, radiation environment of up to $1.3 \times 10^{14} \mathrm{n}_{e q} /$ year.

There are two types of VELO sensors which are shown in Figure 2. The $\mathrm{R}$ sensor has concentric circular strips for radial measurements. The $\Phi$ sensor is segmented in inner and outer radial sections to measure an approximate azimuthal angle, the strips have a stereo angle to provide an additional measurement of the radial position and to solve any possible ambiguities in the tracking. The read out chips are positioned beyond the outer radius of the silicon sensors to minimise the material through which a particle will pass. The signal is routed to the read out chips via a second metal layer. The two types of single sided sensors will be mounted back-to-back on one module. In each silicon station there is a left 


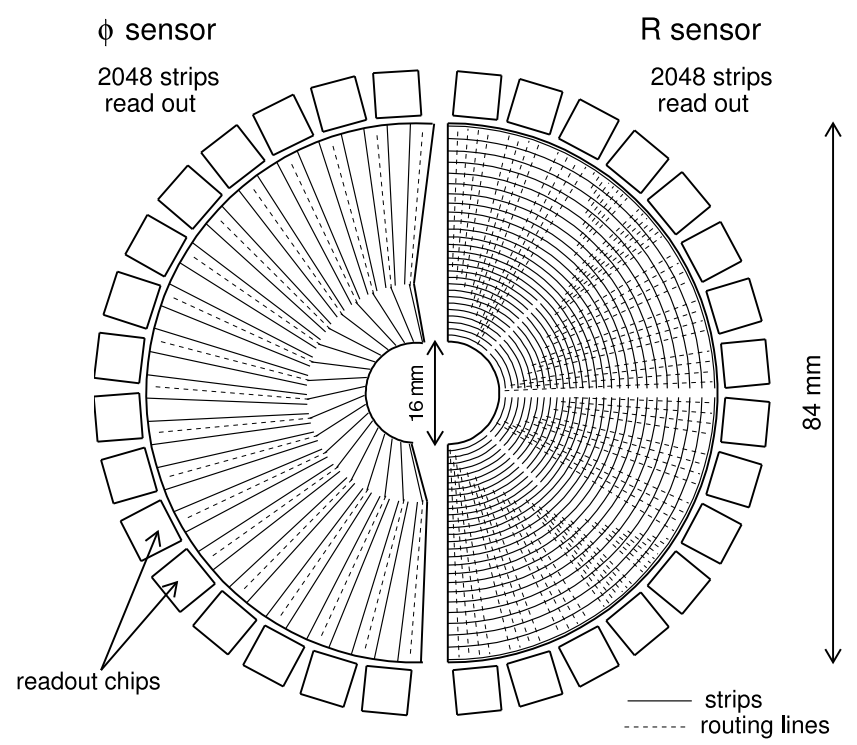

Figure 2: The VELO sensor design. Left: $\Phi$-measuring sensor. Right: R-measuring sensor.

and right module.

A significant programme of research and development has been performed to develop a sensor that is capable of providing a good signal/noise performance for several years of LHCb running, after which replacement of the sensors is foreseen. Proto-type silicon sensors have been manufactured and studied in the laboratory and test-beam environments. Among the significant results that have emerged from these studies is the comparison of identical layout $\mathrm{n}^{+}$-on-n and $\mathrm{p}^{+}$-on-n sensors. These studies [1], identified a number of significant disadvantages of the $\mathrm{p}^{+}$-on-n sensors, particularly when it is necessary to operate the sensors below full depletion. The outcome of the research efforts has resulted in the choice of a complex $\mathrm{n}^{+}$-on-n double metal layer oxygenated silicon solution. However, $\mathrm{n}^{+}$-on- $\mathrm{n}$ technology is expensive and restricts the choice of processing companies as it requires double-sided processing. However, as shown in the next section, the $\mathrm{n}^{+}$-on-n VELO sensors fully satisfy all the requirements of the VELO technical design review [1].

\subsection{New test beam results}

The VELO project is now in the last stage of pre-production. The performance of the almost final silicon sensors and front-end electronics are being verified. The first production series of sensors will arrive throughout October 2004 and will be verified in a test beam at the CERN SPS in November 2004.

LHCb will have the capacity to trigger on consecutive LHC events every $25 \mathrm{~ns}$. In order to maintain a high trigger efficiency no more than $30 \%$ of the peak signal can remain in the next event, this is defined as the spillover. The spillover and the signal to noise ratio are two aspects that must be determined from the VELO sensors performance. A beam test has been performed which tested the performance of a $300 \mu \mathrm{m}$ thick R sensor mounted on a near final hybrid and read out with 16 front end ASICs. The aim was to check the fulfilment of a near to final sensor and readout chip to the VELO requirements.

Figure 3 shows the pulse shapes for different applied bias voltages. The maximum signal to noise ratio for this sensor was 18:1. A previous beam test in 2003 [2] showed the signal to noise ratio for a $200 \mu \mathrm{m}$ sensor to be 12:1. Hence, the measured S:N for the $300 \mu \mathrm{m}$ sensor agrees with the scaled S:N measured for the $200 \mu \mathrm{m}$ sensor. The signal left after $25 \mathrm{~ns}$ from the peak of the signal, spillover, was $30 \%$ when a bias voltage of $100 \mathrm{~V}$ was applied. The S:N and spillover were measured in various regions on the detector, corresponding to varying strip length. The results from this beam test showed that this sensor and readout combination fufills the VELO requirements [1], [3]. 


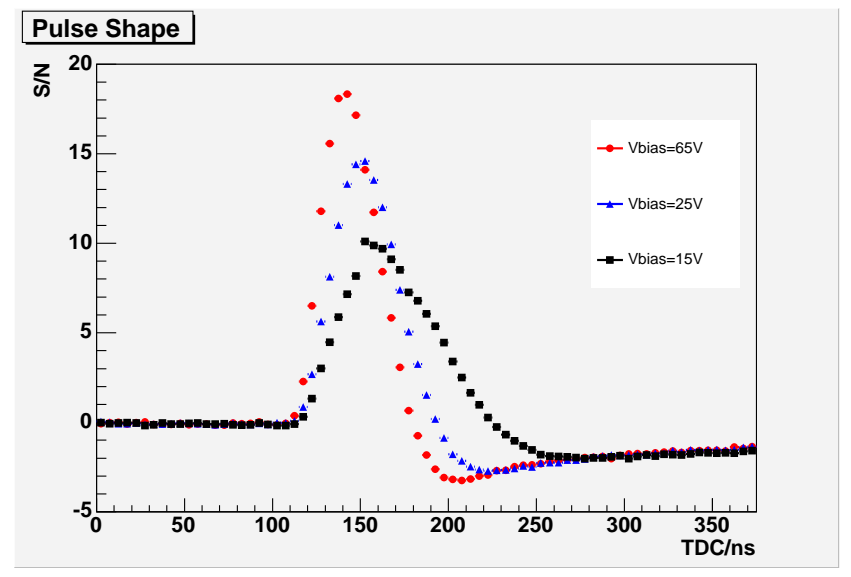

Figure 3: Signal to noise ratio measurements for different applied bias voltages from a $300 \mu \mathrm{m}$ thick $\mathrm{R}$-measuring sensor. The time is in nanoseconds and $\mathrm{t}=0$ is arbitrary.

\section{VELO Upgrade}

\subsection{Current Expectations}

The LHCb VELO provides an extreme challenge for radiation hard silicon studies. Moreover, the irradiation will be strongly non-uniform and will vary from an annual dose of $1.3 \times 10^{14} \mathrm{n}_{e q} / \mathrm{cm}^{2}$ for the smallest radii and sensor closest to the proton interactions to $5 \times 10^{12} \mathrm{n}_{e q} / \mathrm{cm}^{2}$ for the largest radii strip of the sensor furthest away. The VELO detector will operate in one of the most extreme radiation environments of any LHC experiment detector. Currently the $\mathrm{n}^{+}$-on-n oxygenated silicon sensors will survive an estimated three years. A range of different possibilities are available for the VELO upgrade, for example, 3D detectors, pixels or $\mathrm{n}^{+}$-on-p sensors. In this paper the investigations into Magnetic Czochralski (MCz) silicon are reported.

\subsection{Czochralski Silicon}

Recent technological advances have lead to the development of high resistivity magnetic Czochralski silicon. Silicon grown using the $\mathrm{MCz}$ technique has an oxygen content almost an order of magnitude higher than in oxygenated float-zone (DOFZ) grown silicon. The ROSE collaboration showed that increasing the oxygen concentration by diffusion increased the radiation hardness of silicon to charged particle irradiation [4]. Hence the VELO sensors are being produced with DOFZ $\mathrm{n}^{+}$-on-n silicon. However, potentially more manufacturers will make $\mathrm{MCz}$ sensors as only single sided processing is required and an oxygen diffusion process not needed. Indeed even if $\mathrm{MCz}$ does prove to be only as radiation hard as DOFZ, there will still be arguments for moving from DOFZ to $\mathrm{MCz}$ silicon for $\mathrm{HEP}$ experiments. Hence, it is worthwhile investigating this material as a candidate for use in the harsh radiation environments of the LHC.

\subsection{Large $\mathrm{MCz}$ micro strip test beam}

In order to evaluate $\mathrm{MCz}$ as a material for a VELO upgrade, two beam tests using a full scale $\mathrm{MCz}$ micro-strip detector read out at LHC speed, $40 \mathrm{MHz}$, were performed. The $\mathrm{p}^{+}{ }_{-}$-on-n detector had parallel strips of pitch $50 \mu \mathrm{m}$ and the sensor thickness was $380 \mu \mathrm{m}$. The detector was read out with three SCTA analogue chips. One of the beam tests used the unirradiated $\mathrm{MCz}$ detector. The $\mathrm{MCz}$ detector was then non-uniformly irradiated in the CERN PS proton irradiation facility. The maximum fluence received was $7.8 \times 10^{14} 24 \mathrm{GeV} / \mathrm{c} \mathrm{p} / \mathrm{cm}^{2}$. The second beam test analysed the S:N ratio and the charge collection as a function of radiation damage and applied bias voltage. 


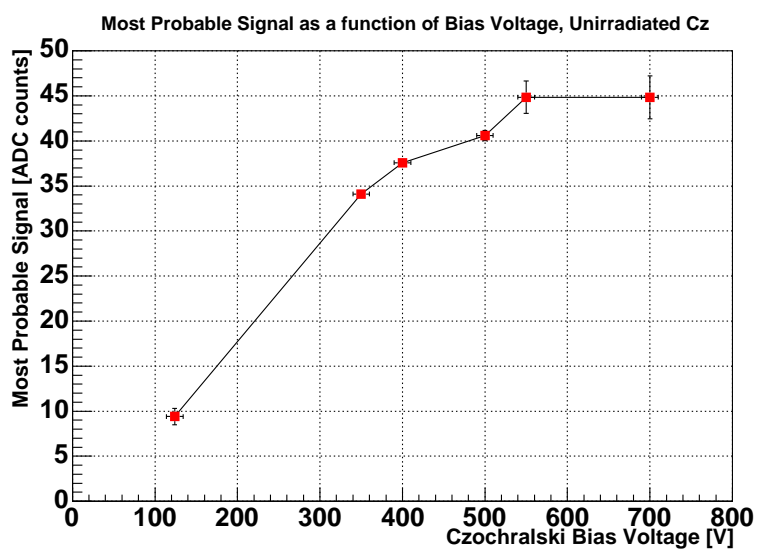

Figure 4: The mean signal measured in ADC counts (uncalibrated) as a function of the applied bias voltage for the $\mathrm{MCz}$ strip detector tested.

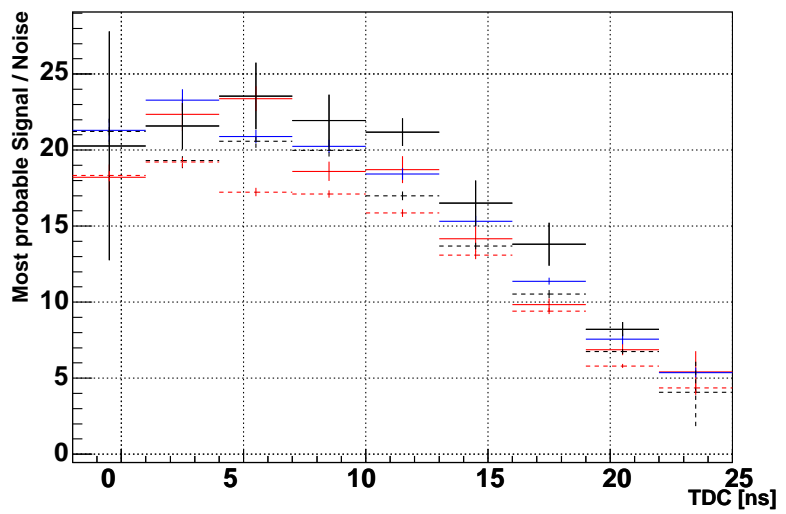

Figure 5: Signal to noise ratio for the unirradiated $\mathrm{MCz}$ detector. the different points refer to different bias voltages applied, from the higher solid points $(700 \mathrm{~V})$ to the lower dashed points $(350 \mathrm{~V})$. the peak signal to noise is estimated at $23.5 \pm 2.5$. 


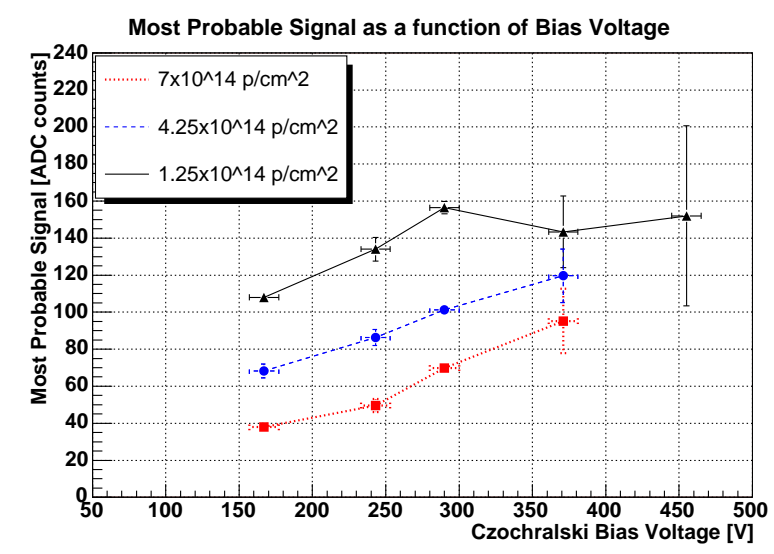

Figure 6: The mean signal measured in ADC counts (uncalibrated) as a function of the applied bias voltage for the $\mathrm{MCz}$ strip detector tested. The radiation levels are quoted for $24 \mathrm{GeV} / \mathrm{c}$ protons $/ \mathrm{cm}^{2}$.

The initial depletion voltage was measured using capacitance-voltage measurements and found to be $420 \mathrm{~V}$. Figure 4 shows a saturation of collected charge at approximately $550 \mathrm{~V}$. The peak signal to noise ratio is approximately $23.5 \pm 2.5$ and is shown in Figure 5. This would fulfil the VELO requirements for initial S:N value.

Figure 6 shows the most probable signal as a function of voltage and irradiation. The unirradiated and irradiated $\mathrm{MCz}$ beam tests were conducted in separate periods hence the ADC settings differed and Figure 4 and Figure 6 can not be directly compared. Unfortunately all the data available for the irradiated $\mathrm{Cz}$ test beam was for voltages less than $500 \mathrm{~V}$. This was due to inadequate cooling, leading to thermal runaway within the $\mathrm{Cz}$ silicon. However, it was still possible to study the effect of radiation on the detector. In the region with the lowest radiation exposure, the collected charge appears to saturate at voltages above $300 \mathrm{~V}$, shown in Figure 6 . The annealing simulation performed for FZ grown silicon resulted in the full depletion voltage for this lowest radiation region to be $150 \mathrm{~V}$ [5]. Hence, the least irradiated region was operated above its full depletion voltage and the two regions with increased radiation damage did not reach full depletion. Note, that due to charge trapping effects, it may be necessary to significantly over-deplete an irradiated detector before the full charge is collected.

Signal to noise values of 15,11 and 7 were found for the three radiation regions which were equivalent to $0.5,2$ and 4 years of the $\mathrm{LHCb}$ radiation environment, respectively. This was measured when the $\mathrm{MCz}$ detector was under-depleted for the highest two of the three radiation regions.

\subsection{Transient Current Technique}

During the irradiation of $\mathrm{p}^{+}$-on-n DOFZ silicon the material undergoes type inversion. The simple explanation of type inversion is when the sign of the space charge changes from positive to negative, which results in the high electric field moving from the structured $\mathrm{p}^{+}$surface to the back surface. Type inversion is caused by donor state removal and acceptor-like states being generated. When such a type-inverted device is operated at a voltage below the full depletion voltage, then there is an non-depleted layer next to the front $\mathrm{p}^{+}$-implants. In this region carriers do not separate and drift under the electric field, only the slow diffusion process occurs. This results in spreading of the charge, hence a loss in resolution.

Studies have been conducted using $300 \mu \mathrm{m}$ thick, $0.25 \mathrm{~cm}^{2} \mathrm{MCz} \mathrm{p}^{+}$-on-n test structures which were produced by the Helsinki Institute of Physics within the framework of RD50 [6]. The test structures were irradiated using the CERN PS $24 \mathrm{GeV} / \mathrm{c}$ proton irradiation facility. The fluence received by the test structure ranges between $(2-50) \times 10^{13} \mathrm{p} / \mathrm{cm}^{2}$. Each test structure has been tested using the Transient Current Technique (TCT). TCT is an experiment which probes the electric field inside the silicon structures through analysing the detector response to photons from a red laser. Photons with a wavelength in the red region are absorbed in silicon within a few micrometers. This results in only one 


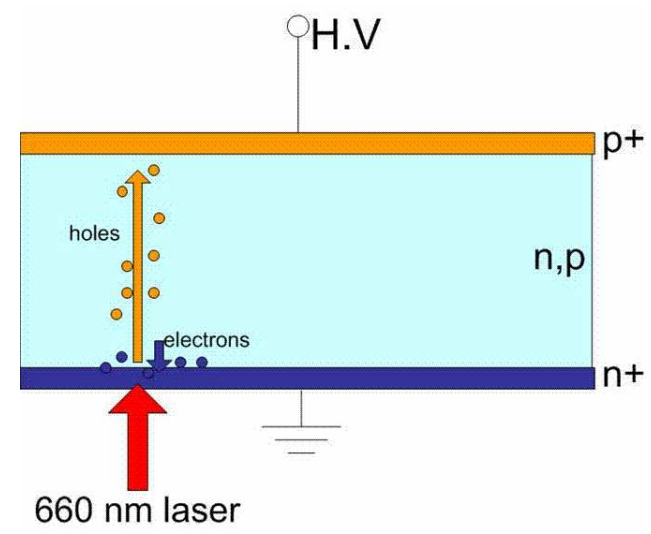

Figure 7: Hole injection: the charge carriers are introduced at the rear $\mathrm{n}^{+}$implant and the holes drift across the whole silicon width (typically $300 \mu \mathrm{m}$ ).

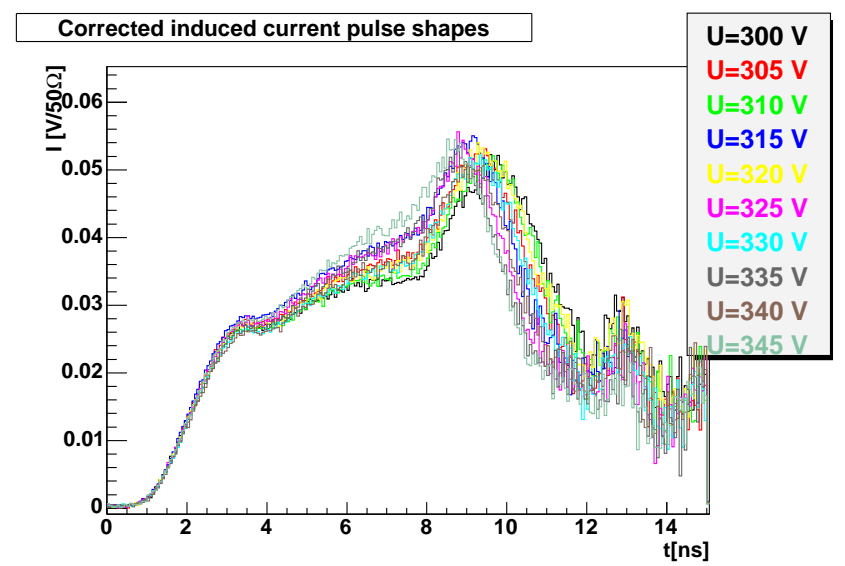

Figure 8: The trapping corrected signal from a $\mathrm{MCz}$ with hole injection. The fluence received by the $\mathrm{MCz}$ detector was $5 \times 10^{14} 24 \mathrm{GeV} / \mathrm{c} \mathrm{p} / \mathrm{cm}^{2}$. The hole are injected at the low field ( $\mathrm{n}^{+}$implant) and move to the high field $\left(\mathrm{p}^{+}\right.$implant)

type of carrier, either electron or hole, drift current dominating the signal. If the laser illuminates the back $\mathrm{n}^{+}$side then hole current will dominate and if the laser illuminates the front $\mathrm{p}^{+}$side then electron current will dominate, see Figure 7.

The movement of the charge carriers instantaneously induces a current. If the current is measured as a function of time, without electronic shaping of the signal, the exact electric field across the silicon can be probed. For example if hole injection is performed, as in Figure 7, then the holes move from the back of the silicon structure to the front $\mathrm{p}^{+}$implant. The instantaneous induced current is proportional to the hole drift velocity, which is proportional to the electric field. Hence TCT can map the electric field within the silicon diode.

TCT was performed for all of the $\mathrm{MCz}$ test structures and it was found that $\mathrm{MCz}$ silicon does not type invert up to $5 \times 10^{14} 24 \mathrm{GeV} / \mathrm{c} \mathrm{p} / \mathrm{cm}^{2}$. This should be compared to identically processed $\mathrm{FZ}$ and DOFZ structures which had type inverted at a dose more than a factor two lower. Figure 8 shows the trapping-corrected signal obtained from illuminating the rear $\mathrm{n}^{+}$side of an $\mathrm{MCz}$ silicon detector and creating a hole dominated signal. The signal is collected over $12 \mathrm{~ns}$ and suffers from noise due to the offline trapping correction procedure. The hole current is low at the time of injection at the back $\mathrm{n}^{+}$ implant. As the holes travel towards the front $\mathrm{p}^{+}$implant there is an increase in their velocity, hence the induced current increases. This can be seen by the positive gradient on the current signal in Figure 8. This proves that even after a fluence of $5 \times 10^{14} 24 \mathrm{GeV} / \mathrm{c} \mathrm{p} / \mathrm{cm}^{2}, \mathrm{p}^{+}$-on-n $\mathrm{MCz}$ does not type invert and the high field remains at the front $\mathrm{p}^{+}$structured side. 
If this result can be reproduced in further experiments then the VELO $\mathrm{n}^{+}$-on-n DOFZ silicon sensors could be replaced by standard $\mathrm{p}^{+}$-on-n $\mathrm{MCz}$ silicon. Research on the comparative radiation hardness of $\mathrm{MCz}$ compared to DOFZ silicon is continuing.

\section{Conclusion}

A significant programme of research and development has been performed to develop a sensor that is capable of providing a good signal/noise performance for several years of LHCb running. The outcome of the research efforts has resulted in the choice of a complex $\mathrm{n}^{+}$-on- $\mathrm{n}$ double metal layer oxygenated silicon solution. The LHCb VELO is now in the final stages of proto-typing and verifying the near final $\mathrm{n}^{+}-$on- $\mathrm{n}$ DOFZ single sided sensors. The first production module will be delivered in October 2004 and tested in a beam test in November. New results from a beam test performed in May 2004 proved that the S:N ratio and spillover levels of a $300 \mu \mathrm{m} R$ measuring sensor are sufficient. Due to the extreme radiation environment of the VELO detector, an upgrade of the sensors is foreseen after approximately three years of operation. One possible upgrade choice, namely Magnetic Czochralski silicon, has been discussed. A beam test of a micro-strip MCz detector showed an acceptable S:N ratio prior to irradiation and revealed the effect of radiation. TCT results on $\mathrm{MCz}$ test structures proved $\mathrm{MCz}$ silicon does not type invert up to a radiation level of $5 \times 10^{14} 24 \mathrm{GeV} / \mathrm{c} \mathrm{p} / \mathrm{cm}^{2}$. This would mean the VELO could replace the complicated processing of $\mathrm{n}^{+}$-on- $\mathrm{n}$ DOFZ sensors by standard $\mathrm{p}^{+}$-on- $\mathrm{MCz}$, however, further investigation of the radiation tolerance of $\mathrm{MCz}$ is required.

\section{Acknowledgment}

The authors would like to thank Maurice Glaser, Federico Ravotti and Michael Moll for the irradiations of the $\mathrm{MCz}$ detector in May 2003. We also thank to Jaakko Haerkoenen and the Helsinki Institute of Physics for providing the VELO with the MCz detector and the test structures for the TCT studies. The TCT work was developed between the VELO group and the RD50 collaboration, particular thanks to Michael Moll.

\section{References}

[1] LHCb Collaboration, LHCb Vertex Locator Technical Report, CERN/LHCC/2001-011 (2001)

[2] J. Buytaert, P. Collins, D. Eckstein, J. P. Palacios, "Performance of a Beetle1.2 chip reading out a Micron PR03 R measuring sensor", LHCb-2003-135, June 04.

[3] A. Bates, J Buytaert, L. Eklund, D. Eckstein, J. P. Palacios, N. A. Smith, et al., "Pulseshape characteristics of a $300 \mu \mathrm{m}$ sensor read out through a Beetle1.3 chip", LHCb-2004-068, September 04.

[4] RD48 (ROSE) collaboration, "Radiation hard silicon detectors - developments by the RD48 (ROSE) collaboration", Nucl. Instrum. Methods in Phys. Res. A 466, 308-326, 2001.

[5] A. G. Bates, J. Buytaert, P. Collins, D. Eckstein, J. Kennedy, T Ketel, J. P. Palacios, C Parkes, U Parzefall, I Stavitski, N Tuning, "Results from the first test beam of a large microstrip Czochralski silicon detector equipped with LHC electronics", LHCb-2004-052, June 04.

[6] M. Moll on behalf of the RD50 collaboration, "Development of radiation hard sensors for very high luminosity colliders - CERN-RD50 project", Nucl. Instrum. Methods in Phys. Res. A 511, 1-2, 97-105, 2003. 